\section{Influência do excesso de peso e da obesidade nos gastos em saúde nos domicílios brasileiros}

\author{
The influence of excess weight and obesity on \\ health spending in Brazilian households
}

\author{
Influencia del exceso de peso y la obesidad en el \\ gasto en salud en los hogares brasileños
}

\author{
1 Faculdade de Saúde \\ Pública, Universidade de São \\ Paulo, São Paulo, Brasil. \\ 2 Faculdade de Medicina, \\ Universidade de São Paulo, \\ São Paulo, Brasil. \\ Correspondência \\ D. S. Canella \\ Programa de Pós-graduação \\ em Nutrição em Saúde \\ Pública, Faculdade de Saúde \\ Pública, Universidade de São \\ Paulo. \\ Av. Dr. Arnaldo 715, São Paulo, \\ SP 01246-904, Brasil. \\ dcanella@usp.br
}

\begin{abstract}
The objective of this study was to evaluate the influence of excess weight and obesity on health spending in Brazilian households. Data from the Household Budget Survey 2008-2009 were used to estimate monetary health spending, corresponding to out-of-pocket spending, including purchase of medicines and payment for healthcare services, and to evaluate the nutritional status of the 55,970 household residents. Monthly spending on health and its components were analyzed according to the number of excess weight and obese individuals in households (none, one, two, or three or more individuals). The presence and increasing number of excess weight and obese individuals has resulted in greater spending on health, especially on medicines and health insurance. The results were maintained after adjusting for income, region, area, and presence of elderly and number of residents in the household. Excess weight and obesity had a direct impact on outof-pocket health spending by Brazilian families.

Obesity; Health Expenditures; Drugs;

Delivery of Health Care
\end{abstract}

Daniela Silva Canella 1 Hillegonda Maria Dutilh Novaes 2 Renata Bertazzi Levy 2

\section{Resumo}

O objetivo deste estudo foi avaliar a influência do excesso de peso e da obesidade sobre os gastos em saúde nos domicílios brasileiros. Dados da Pesquisa de Orçamentos Familiares de 2008-2009 foram utilizados para estimar os gastos familiares privados em saúde, que compreendem os gastos por desembolso direto, incluindo a aquisição de medicamentos $e$ o pagamento por serviços de assistência à saúde, $e$ avaliar o estado nutricional dos moradores dos 55.970 domicílios. Os gastos mensais em saúde e seus componentes foram analisados segundo o número de indivíduos com excesso de peso $e$ obesidade nos domicílios (nenhum, um, dois ou três, ou mais indivíduos). A presença e o aumento do número de indivíduos com excesso de peso e obesidade resultaram em maiores gastos em saúde, com destaque para as despesas com medicamentos e planos de saúde. Tais resultados foram mantidos após ajuste (renda, região, área, presença de idosos e número de moradores nos domicílios). $O$ excesso de peso e a obesidade influenciaram positivamente os gastos privados em saúde das famílias brasileiras.

Obesidade; Gastos em Saúde; Medicamentos; Assistência à Saúde 


\section{Introdução}

A obesidade é uma doença de complexa e múltipla determinação, que atinge a maior parte dos países do mundo, independentemente destes serem desenvolvidos ou em desenvolvimento 1,2 . Dados do último inquérito que avaliou o estado nutricional da população brasileira indicam que a prevalência de obesidade entre crianças, adolescentes e indivíduos maiores de 20 anos é de, respectivamente, $14,3 \%, 4,9 \%$ e $14,8 \%{ }^{3}$. Como consequência, a obesidade resulta em elevado custo para diversos países, principalmente aqueles desenvolvidos 4, e no Brasil já se conhece a magnitude do impacto do sobrepeso e da obesidade para o sistema público de saúde, o Sistema Único de Saúde (SUS) 5,6,7.

O SUS foi instituído pela Constituição Federal de 1988 e é um sistema de atenção à saúde universal, baseado nos princípios da saúde como um direito do cidadão e um dever do Estado. No entanto, o sistema de saúde brasileiro ultrapassa os limites do SUS, sendo formado por uma rede complexa de prestadores e compradores de serviços que competem entre si, gerando uma combinação público-privada ${ }^{8}$. Nesse sentido, a obtenção de medicamentos e a utilização de serviços de saúde pode se dar por distintas formas de financiamento: o SUS, ou seja, o sistema público de saúde; a saúde suplementar, com os planos e seguros privados de saúde; e a compra de produtos e serviços por desembolso direto (out-of-pocket) 9 .

De acordo com dados do Instituto Brasileiro de Geografia e Estatística (IBGE), 53\% dos dispêndios nacionais com saúde se referem ao gasto das famílias, enquanto o setor público arca com $46 \%$ do total gasto com saúde no país. Assim, apesar do SUS ter caráter universal, expressiva parcela do dispêndio nacional com saúde é resultado do pagamento por produtos e serviços de saúde pelas famílias brasileiras 10 . Dessa forma, estudos sobre esses gastos e fatores que os influenciam mostram-se relevantes. Os gastos privados em saúde das famílias podem levar à redução de outras despesas essenciais do domicílio durante um período, curto ou prolongado, para enfrentar estes dispêndios adicionais em saúde, além de poder levá-las a uma situação de endividamento ou falência, condição que tem sido denominada de empobrecimento devido a gastos catastróficos em saúde, que ocorre mais frequentemente em países com renda média ou baixa ${ }^{11}$. Além dos gastos médicos diretos, com procedimentos e medicamentos, podem ocorrer gastos indiretos, com perda de produtividade, dispêndios com cuidadores e, caso o problema de saúde afete o responsável pela renda familiar, pode ocorrer a diminuição ou mesmo ausência de renda 12 .

Achados da literatura nacional e internacional apontam como fatores que afetam potencialmente os gastos familiares em saúde: renda domiciliar 13,14,15 e localização dos domicílios (região e área) 16, ambas relacionadas ao acesso à saúde; mas também a composição familiar, como a presença de idoso nos domicílios 13,14,17 e a ocorrência de determinadas doenças entre os moradores, com destaque para as condições crônicas 15,18,19. A obesidade também está relacionada a alguns desses fatores 20,21,22 e, uma vez que esta tem impacto financeiro sobre o SUS $5,6,7$, pode também resultar em maiores gastos em saúde para as famílias.

Considerando que uma parcela superior a $50 \%$ dos dispêndios em saúde no Brasil decorrem do pagamento por serviços e produtos pelas famílias, que apenas o custo da obesidade para o SUS é conhecido e que a presença de obesos nos domicílios pode afetar os gastos familiares em saúde, faz-se necessário explorar a influência da obesidade sobre o orçamento das famílias brasileiras. Nesse sentido, o objetivo do presente trabalho foi avaliar a influência do excesso de peso e da obesidade sobre os gastos em saúde nos domicílios brasileiros, utilizando os dados da Pesquisa de Orçamentos Familiares (POF) 2008-2009.

\section{Métodos}

Os dados utilizados são provenientes da POF, conduzida pelo IBGE, no período de 19 de maio de 2008 a 18 de maio de 2009 (POF 2008-2009).

$\mathrm{O}$ estudo tem representatividade nacional e envolveu 55.970 domicílios brasileiros, selecionados com base em um plano amostral complexo, por conglomerados em dois estágios, com o sorteio dos setores censitários no primeiro estágio e de domicílios no segundo. Os setores censitários foram selecionados a partir de amostra comum ou amostra mestra do IBGE, que envolve setores agrupados a fim de obter estratos de domicílios com alta homogeneidade geográfica e socioeconômica. Para a construção dos estratos foram consideradas a localização geográfica do setor (região; estado; situação urbana ou rural; e municípios da capital, da região metropolitana e demais municípios) e, internamente, em cada lócus geográfico, o espectro de variação socioeconômica das famílias residentes (com base na renda do indivíduo responsável pelo domicílio). A seleção da subamostra de setores censitários para a POF 2008-2009 foi realizada por amostragem aleatória simples em cada estrato. As uni- 
dades secundárias de amostragem foram os domicílios particulares permanentes, selecionados por amostragem aleatória simples sem reposição, dentro de cada um dos setores censitários selecionados. Finalmente, os setores censitários foram distribuídos ao longo dos quatro trimestres da pesquisa, garantindo que em todos os trimestres os estratos estivessem representados pelos domicílios selecionados, a fim de contemplar as alterações a que estão sujeitas as despesas ao longo do ano 23.

Ademais, tal procedimento de amostragem permite a obtenção de estimativas representativas do conjunto de domicílios do país como um todo, das cinco regiões, das 27 Unidades da Federação, das nove regiões metropolitanas e das capitais dos estados, em relação à situação urbana, e, no caso do país e das grandes regiões, permite, ainda, estimativas representativas para as áreas urbana e rural 23.

As informações da POF 2008-2009 utilizadas no presente estudo se referem aos gastos familiares privados em saúde, incluindo a aquisição de medicamentos e o pagamento por planos de saúde e serviços de assistência à saúde, e ao estado nutricional dos moradores dos domicílios estudados. Adicionalmente, utilizaram-se dados referentes à renda domiciliar per capita e às características demográficas dos domicílios (região e área).

Os dados referentes aos gastos em saúde foram informados, em questionário específico sobre despesas individuais, por cada um dos membros do domicílio maiores de 10 anos. Além dos vários itens já presentes no questionário, referentes à aquisição de produtos farmacêuticos e pagamento por serviços de assistência à saúde, itens não listados poderiam ser incluídos pelos entrevistados.

Ainda, foram coletadas informações sobre a forma de aquisição dos medicamentos (monetária ou não monetária). Neste trabalho foram consideradas apenas as despesas monetárias, que compreendem os gastos em saúde por desembolso direto (out-of-pocket) e o pagamento de planos e seguros de saúde, portanto, utilizouse a informação sobre a forma de aquisição, a fim de excluir despesas não monetárias. Em seguida, calculou-se o valor deflacionado dos gastos, em Reais (R\$), usando-se o Índice Nacional de Preços ao Consumidor Amplo (IPCA), tendo como data de referência o dia 15 de janeiro de 2009, a fim de corrigir as mudanças absolutas e relativas nos preços que ocorrem ao longo do ano 23 .

Apesar dos gastos em saúde terem sido referidos no nível individual, optou-se por considerar o domicílio como unidade de análise, uma vez que gastos desta natureza raramente são estrita- mente individuais 24 , independentes da dinâmica familiar 25, e pelo fato de apenas os indivíduos maiores de 10 anos reportarem os gastos, sendo que os menores tiveram seus dispêndios computados por outro morador do domicílio.

Como o período de referência para o conjunto de gastos em saúde não era o mesmo (30 dias para medicamentos e 90 dias para serviços de assistência à saúde), calculou-se os gastos mensais com assistência à saúde. Por fim, os gastos em saúde foram agrupados em gastos com medicamentos (para doenças crônicas, para doenças infecciosas e outros) e com assistência à saúde (planos de saúde, internações, consultas, exames e outros).

Em relação à avaliação do estado nutricional, consideraram-se todos os indivíduos residentes nos domicílios estudados ( $\mathrm{n}=190.159)$. As medidas de peso e altura foram aferidas por agente de pesquisa, por meio de técnicas padronizadas, e registradas em questionário específico referente às características dos moradores e do domicílio. Para a mensuração do peso, registrado em quilogramas, foi utilizada balança eletrônica portátil, com capacidade de $150 \mathrm{~kg}$ e graduação de $100 \mathrm{~g}$. A altura, registrada em centímetros, compreende a medida do comprimento em crianças de 0 a 23 meses de idade e da estatura em indivíduos a partir de 24 meses. Para mensurar o comprimento, foram utilizados antropômetros infantis com capacidade de até $105 \mathrm{~cm}$, com escala milimétrica, e a estatura foi medida utilizando-se estadiômetros portáteis, com trena retrátil, de extensão até $200 \mathrm{~cm}$ e com precisão de $0,1 \mathrm{~cm}^{3}$.

Baseando-se nas medidas de peso e altura aferidas, foram calculados o índice de massa corporal (IMC) para adultos e idosos e o IMC-paraidade para crianças e adolescentes, expresso em escore-Z. A classificação do estado nutricional dos indivíduos seguiu as recomendações da Organização Mundial da Saúde (OMS) para cada faixa etária. Crianças menores de cinco anos foram classificadas como tendo excesso de peso quando apresentaram escore- $Z$ maior que $+2 \mathrm{e}$ obesidade em caso de escore- $Z$ maior que +326 . Entre as crianças com cinco ou mais anos e adolescentes (até 19 anos), excesso de peso e obesidade foram caracterizados por escore- $Z$ maior que +1 e escore- $Z$ maior que +2 , respectivamente 27 . Para adultos e idosos um IMC maior ou igual a $25 \mathrm{~kg} / \mathrm{m}^{2}$ foi classificado como excesso de peso e maior ou igual a $30 \mathrm{~kg} / \mathrm{m}^{2}$ como obesidade 28 .

Tendo os domicílios como unidade de estudo, a partir da classificação do estado nutricional dos moradores de cada domicílio foram construídas as variáveis "número de indivíduos com excesso de peso nos domicílios" e "número de indivíduos obesos nos domicílios", ambas tendo como cate- 
gorias "nenhum indivíduo", "um indivíduo”, “dois indivíduos" e "três ou mais indivíduos".

O número de moradores dos domicílios com excesso de peso e obesidade e os gastos familiares em saúde e seus componentes foram descritos segundo variáveis que potencialmente afetam os gastos familiares em saúde, como renda domiciliar mensal per capita (expressa em terços), região, área e presença de idosos nos domicílios 13,14,15,16,17.

Os gastos em saúde mensais (medicamentos, assistência à saúde e gasto total) foram descritos segundo o estado nutricional dos moradores dos domicílios (número de indivíduos com excesso de peso e número de indivíduos obesos nos domicílios). Testou-se o significado estatístico das diferenças para cada um dos componentes dos gastos em saúde, segundo o número de indivíduos com excesso de peso e obesos nos domicílios, por teste de comparação de médias. Adicionalmente, foram calculados os valores esperados (valores preditos por modelos de regressão) de gastos em saúde, ajustados por renda domiciliar per capita, região, área, presença de idosos e número de moradores nos domicílios, segundo o número de indivíduos com excesso de peso e número de indivíduos obesos nos domicílios. A diferença entre os valores preditos foi comparada por meio de modelos de regressão linear múltipla, tendo como desfecho cada um dos componentes do gasto em saúde e como exposição a contagem de indivíduos com excesso de peso e obesidade nos domicílios, sendo a condição de ausência de indivíduo com excesso de peso ou obesidade considerada a categoria de referência.

Todas as análises foram realizadas com o emprego do pacote estatístico Stata/SE versão 12.1 (StataCorp LP, College Station, Estados Unidos), no módulo survey, que considera os efeitos da amostragem complexa e permite a extrapolação dos resultados para a população brasileira, foi considerado intervalo de $95 \%$ de confiança e nível de significância de $5 \%$.

\section{Resultados}

Foram estudados 55.970 domicílios brasileiros. Desses, apenas cerca de $24 \%$ não tinham nenhum indivíduo com excesso de peso em sua composição, e cerca de 13\% dos domicílios apresentavam três ou mais indivíduos com excesso de peso. Em relação à obesidade, $32 \%$ dos domicílios tinham ao menos um indivíduo obeso em sua composição. Domicílios com moradores obesos foram observados em maior frequência nas regiões Sul e Sudeste, na área urbana, nas faixas de renda média e alta (Tabela 1).
Os gastos privados em saúde dos domicílios brasileiros foram, em média, $\mathrm{R} \$ 249,53$ mensais, sendo $23 \%$ destinados à aquisição de medicamentos e $55 \%$ ao pagamento de planos de saúde. Entre os medicamentos, os maiores valores gastos foram observados no centro-sul do país, na área urbana, nos domicílios mais ricos e com idosos em sua composição. Cenário semelhante foi verificado para os dispêndios com assistência à saúde. Os valores foram superiores na Região Sudeste, na área urbana, entre os domicílios com idosos e com maior renda domiciliar. Destacase que no estrato de renda inferior, nas regiões Norte e Nordeste e na área rural os medicamentos apresentaram uma maior participação nos gastos em saúde, chegando a representar, no estrato de renda inferior, quase $50 \%$ destes gastos (Tabela 2).

Entre os domicílios estudados, $17 \%$ não referiram gastos com medicamentos no período de referência da pesquisa. Em relação à assistência à saúde, $64 \%$ não apresentaram quaisquer gastos no período, sendo que apenas $18 \%$ referiram dispêndios com plano de saúde, 13\% com consultas e $12 \%$ com internação. Ainda, os gastos privados em saúde comprometeram $4,6 \%$ da renda domiciliar per capita, sendo que os gastos com medicamentos comprometeram $3,4 \%$ da renda. Considerando-se os diferentes níveis de renda, os dispêndios em saúde comprometeram $6,0 \%$ da renda da população mais pobre (1o terço de renda), $4,4 \%$ para a população com nível intermediário de renda (2o terço) e entre os mais ricos (3o terço), o comprometimento foi de $3,4 \%$.

Verificou-se que a presença e o aumento do número de indivíduos com excesso de peso e de obesidade nos domicílios resultaram em maiores gastos em saúde, sugerindo um efeito "doseresposta” em relação ao aumento do número de indivíduos nestas condições nos domicílios. Destaca-se o aumento nos dispêndios com medicamentos (para doenças crônicas, doenças infecciosas) e com assistência à saúde, influenciado pelas despesas com planos de saúde. Quando foram analisados os gastos com assistência à saúde excluindo-se as despesas com planos de saúde, não houve diferença significativa em relação aos domicílios com e sem indivíduos com excesso de peso e obesidade. Ainda que os valores absolutos de gastos com internação sugiram um aumento do gasto em função do número de indivíduos com excesso de peso e obesidade, esta diferença não foi estatisticamente significativa (Tabela 3).

Dada a relação direta entre renda e gastos em saúde observada, foram feitas análises excluindo-se os domicílios com valores extremos de renda mensal per capita (inferior a $\mathrm{R} \$ 210,00$, 
Distribuição dos domicílios brasileiros de acordo com o número de indivíduos com excesso de peso e obesidade em sua composição, segundo variáveis que potencialmente afetam os gastos em saúde. Pesquisa de Orçamentos Familiares, Brasil, 2008-2009.

\begin{tabular}{|c|c|c|c|c|c|c|c|c|}
\hline \multirow[t]{2}{*}{$\begin{array}{l}\text { Características } \\
\text { sociodemográficas }\end{array}$} & \multicolumn{4}{|c|}{$\begin{array}{c}\text { Proporção de domicílios segundo número } \\
\text { de indivíduos com excesso de peso em sua } \\
\text { composição (\%) }\end{array}$} & \multicolumn{4}{|c|}{$\begin{array}{l}\text { Proporção de domicílios segundo número de indivíduos } \\
\text { obesos em sua composição (\%) }\end{array}$} \\
\hline & Nenhum & 1 & 2 & 3 ou mais & Nenhum & 1 & 2 & 3 ou mais \\
\hline \multicolumn{9}{|c|}{$\begin{array}{l}\text { Renda domiciliar mensal } \\
\text { per capita (R\$) }\end{array}$} \\
\hline 1ㅇ terço $(220,68)$ & 24,8 & 35,8 & 24,5 & 14,9 & 68,8 & 24,5 & 5,5 & 1,1 \\
\hline 2o terço $(585,42)$ & 23,6 & 37,2 & 26,5 & 12,8 & 67,2 & 25,5 & 5,9 & 1,3 \\
\hline 3o terço $(2.346,52)$ & 22,8 & 41,2 & 25,3 & 10,8 & 67,6 & 26,2 & 5,3 & 0,9 \\
\hline \multicolumn{9}{|l|}{ Região } \\
\hline Norte & 24,5 & 35,8 & 24,9 & 14,7 & 68,8 & 25,0 & 5,4 & 0,9 \\
\hline Nordeste & 27,3 & 37,7 & 23,2 & 11,8 & 71,1 & 23,5 & 4,5 & 1,0 \\
\hline Sudeste & 22,3 & 38,8 & 26,1 & 12,8 & 67,2 & 25,9 & 5,8 & 1,2 \\
\hline Sul & 20,4 & 37,4 & 27,8 & 14,4 & 63,8 & 27,7 & 7,1 & 1,5 \\
\hline Centro-oeste & 25,7 & 38,3 & 24,6 & 11,4 & 68,9 & 25,1 & 5,3 & 0,8 \\
\hline \multicolumn{9}{|l|}{ Área } \\
\hline Rural & 28,3 & 38,9 & 22,3 & 10,5 & 71,4 & 23,5 & 4,5 & 0,6 \\
\hline Urbana & 22,9 & 37,9 & 26,0 & 13,2 & 67,3 & 25,8 & 5,8 & 1,2 \\
\hline $\begin{array}{l}\text { Presença de idosos (6 } \\
\text { anos e mais) no domic }\end{array}$ & \multicolumn{8}{|c|}{ Presença de idosos (65 } \\
\hline Não & 24,0 & 37,7 & 25,6 & 12,7 & 68,5 & 24,8 & 5,5 & 1,2 \\
\hline Sim & 22,8 & 39,4 & 24,6 & 13,1 & 65,4 & 27,8 & 5,9 & 0,9 \\
\hline Brasil & 23,7 & 38,1 & 25,4 & 12,8 & 67,9 & 25,4 & 5,6 & 1,1 \\
\hline
\end{tabular}

referente ao critério para participação no Programa Bolsa Família no ano de 2009, e superior a R\$ 6.121,00 que corresponde à renda per capita da parcela mais rica da população - 1\%). Como não foram observadas alterações nos resultados, optou-se por apresentar apenas as análises com todos os domicílios estudados.

Adicionalmente, foram calculados os valores preditos para os gastos em saúde e seus componentes, ajustados por renda domiciliar per capi$t a$, região, área, presença de idosos e número de moradores nos domicílios, variáveis associadas aos gastos em saúde, em função do número de indivíduos com excesso de peso e obesidade nos domicílios. Observou-se elevação dos gastos em saúde devido à presença e aumento do número de indivíduos com excesso de peso e obesidade nos domicílios, sendo que a magnitude do aumento dos gastos foi maior para a obesidade, mesmo após ajuste. Quanto à obesidade, as únicas diferenças observadas em relação à análise sem ajuste foram para os gastos com "outros medicamentos" e com "assistência à saúde", ainda que este último estivesse no limite da significân- cia $(p=0,06)$. Comparados a domicílios sem nenhum indivíduo obeso em sua composição, ter um indivíduo obeso no domicílio resultou em $15 \%$ a mais nos gastos, ter dois obesos em $40 \%$ a mais e ter três ou mais resultou em um gasto em saúde $195 \%$ superior (Tabela 4).

\section{Discussão}

Com base em dados representativos da população brasileira, verificou-se que em 2008-2009 a proporção de domicílios com indivíduos com excesso de peso e obesidade em sua composição e os gastos familiares privados em saúde foram maiores no centro-sul, na área urbana do país e entre os domicílios com maior renda. A presença e o aumento do número de indivíduos com excesso de peso e obesidade, com maior magnitude para este último, resultaram em maior dispêndio total em saúde, com destaque para os gastos com medicamentos e planos de saúde, mesmo após ajuste para as variáveis de confusão associadas aos gastos e ao estado nutricional dos 
Tabela 2

Distribuição dos gastos familiares em saúde mensais (medicamentos e assistência à saúde), segundo variáveis que potencialmente afetam os gastos em saúde. Pesquisa de Orçamentos Familiares, Brasil, 2008-2009.

\begin{tabular}{|c|c|c|c|c|c|c|c|c|c|c|c|}
\hline \multirow[t]{3}{*}{ Variáveis } & \multicolumn{11}{|c|}{ Gastos familiares em saúde mensais (Reais) } \\
\hline & \multicolumn{4}{|c|}{ Medicamentos } & \multicolumn{6}{|c|}{ Assistência à saúde } & \multirow[t]{2}{*}{ Total } \\
\hline & $\begin{array}{l}\text { Para } \\
\text { doenças } \\
\text { crônicas }\end{array}$ & $\begin{array}{c}\text { Para } \\
\text { doenças } \\
\text { infecciosas }\end{array}$ & Outros & Total & $\begin{array}{l}\text { Planos } \\
\text { de } \\
\text { saúde }\end{array}$ & Internações & Consultas & Exames & Outros & Total & \\
\hline \multicolumn{12}{|l|}{ Renda } \\
\hline \multicolumn{12}{|l|}{ domiciliar } \\
\hline \multicolumn{12}{|l|}{ per capita } \\
\hline $1^{\circ}$ terço & 9,80 & 13,30 & 6,60 & 29,70 & 10,56 & 13,20 & 7,52 & 0,34 & 0,22 & 31,85 & 61,55 \\
\hline $2^{\circ}$ terço & 21,37 & 17,52 & 10,67 & 49,56 & 51,93 & 22,02 & 16,15 & 0,55 & 0,77 & 91,42 & 140,98 \\
\hline $3^{\circ}$ terço & 46,11 & 23,50 & 20,71 & 90,32 & 347,13 & 52,00 & 51,53 & 0,75 & 4,32 & 455,73 & 546,05 \\
\hline \multicolumn{12}{|l|}{ Região } \\
\hline Norte & 11,42 & 19,22 & 10,43 & 41,06 & 44,45 & 19,66 & 13,11 & 0,63 & 0,62 & 78,47 & 119,53 \\
\hline Nordeste & 15,35 & 13,91 & 9,76 & 39,02 & 67,79 & 19,35 & 13,23 & 0,72 & 0,64 & 101,72 & 140,74 \\
\hline Sudeste & 31,37 & 18,78 & 14,41 & 64,57 & 209,98 & 27,17 & 33,49 & 0,40 & 2,31 & 273,36 & 337,94 \\
\hline Sul & 33,80 & 22,35 & 14,02 & 70,17 & 108,54 & 51,86 & 28,63 & 0,42 & 1,72 & 191,17 & 261,34 \\
\hline $\begin{array}{l}\text { Centro- } \\
\text { oeste }\end{array}$ & 25,60 & 19,05 & 11,70 & 56,35 & 86,14 & 35,86 & 20,39 & 1,01 & 3,64 & 147,03 & 203,38 \\
\hline \multicolumn{12}{|l|}{ Área } \\
\hline Rural & 15,65 & 15,76 & 8,12 & 39,52 & 23,28 & 22,58 & 15,37 & 0,74 & 0,54 & 62,51 & 102,03 \\
\hline Urbana & 27,63 & 18,54 & 13,50 & 59,67 & 157,45 & 30,27 & 26,86 & 0,51 & 1,99 & 217,09 & 276,76 \\
\hline \multicolumn{12}{|l|}{ Presença de } \\
\hline \multicolumn{12}{|l|}{ idosos $(65$} \\
\hline \multicolumn{12}{|l|}{ anos e mais) } \\
\hline \multicolumn{12}{|l|}{ no domicílio } \\
\hline Não & 6,57 & 5,88 & 4,25 & 18,36 & 14,52 & 2,89 & 3,25 & 0,06 & 4,39 & 25,11 & 43,46 \\
\hline Sim & 27,31 & 8,30 & 6,43 & 45,83 & 34,09 & 5,08 & 4,65 & 0,08 & 6,98 & 50,87 & 96,70 \\
\hline Brasil & 25,76 & 18,11 & 12,66 & 56,53 & 136,54 & 29,07 & 25,07 & 0,55 & 1,77 & 193,00 & 249,53 \\
\hline
\end{tabular}

indivíduos. Após a correção monetária pelo IPCA dos valores verificados, em 15 de janeiro de 2015, ter três ou mais indivíduos com excesso de peso ou obesidade no domicílio representaria um gasto mensal em saúde de $\mathrm{R} \$ 378,87$ e R \$ 746,91, respectivamente, considerando-se que todos os itens que compuseram os gastos em saúde registraram valorização semelhante.

Diferentemente de trabalhos sobre o custo da obesidade para os sistemas de saúde, como o SUS 5,6, em estudos de gasto privado em saúde não é comum analisar o sobrepeso ou o excesso de peso (sobrepeso + obesidade). Assim, destaca-se, neste estudo, o fato de o sobrepeso ter sido analisado na categoria de "domicílios com indivíduos com excesso de peso”. Ressaltamos a importância dessa análise adicional, uma vez que mesmo a condição de sobrepeso impactou nos gastos familiares em saúde.
Na literatura científica são escassos os trabalhos que avaliam a influência da obesidade sobre os gastos familiares em saúde, sendo mais frequentes aqueles que tratam do seu custo para o sistema de saúde e, entre os estudos localizados, os métodos e variáveis empregados nas análises são diversos. No entanto, os trabalhos disponíveis corroboram com os nossos achados.

Em Portugal, um estudo realizado em 19981999 mostrou que cerca de $2 \%$ dos gastos familiares em saúde foram atribuíveis ao sobrepeso e $3 \%$ à obesidade ${ }^{29}$. Além disso, a despesa adicional de indivíduos com sobrepeso e obesidade em relação àqueles com peso adequado foi de $50 \mathrm{e}$ 80 Euros/per capita/ano, respectivamente. Ainda que os gastos em saúde decorrentes do sobrepeso ou da obesidade sejam pequenos em termos absolutos, estes podem representar um custo significativo para as famílias de baixa renda 29 , 
Distribuição dos gastos familiares em saúde mensais (medicamentos e assistência à saúde), de acordo com o tipo de gasto, segundo o número de indivíduos com excesso de peso e obesidade nos domicílios brasileiros. Pesquisa de Orçamentos Familiares, Brasil, $2008-2009$.

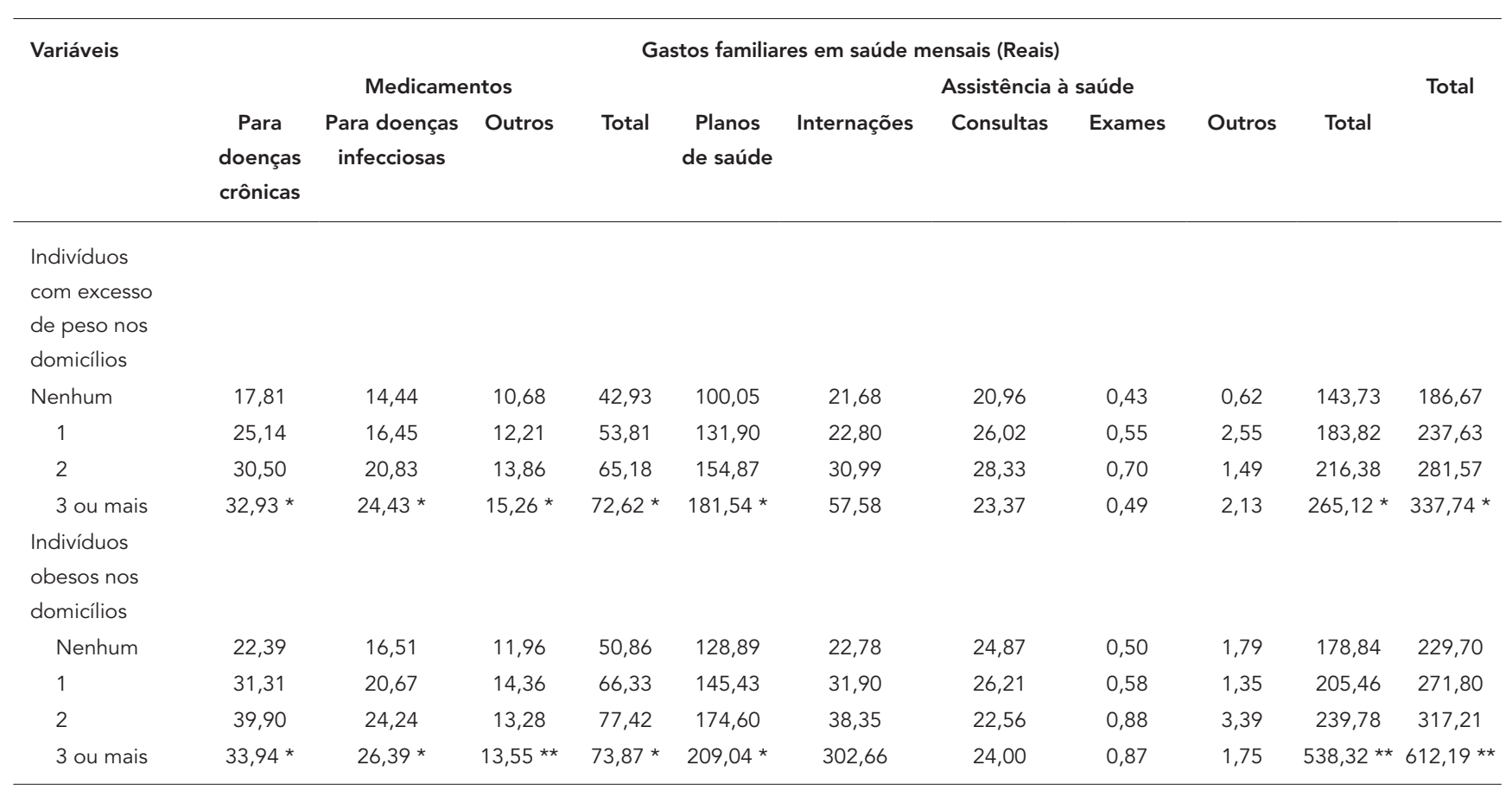

$\star p<0,001$;

** $p<0,05$.

as quais, frequentemente, comprometem maior parte de seu orçamento com a saúde 30.

Para a população norte-americana foram encontradas diferenças significativas com os gastos em saúde de obesos (US\$ 585,44) e não obesos (US\$ 333,24). As diferenças decorreram, principalmente, dos dispêndios com medicamentos 31 , resultado semelhante ao verificado em nosso estudo. Ainda em relação aos norte-americanos, os gastos em saúde cresceram com o aumento do IMC. Adultos obesos apresentaram maiores gastos com medicamentos e com consultas 32 . No Canadá, os dispêndios com internação e com consultas médicas foram, respectivamente, $40 \%$ e $22 \%$ maiores entre os adultos obesos em relação àqueles com peso adequado 33 .

Em estudos que tiveram como exposição uma ou mais doenças crônicas, verificou-se que os domicílios com moradores nesta situação apresentaram maiores gastos em saúde 15,19,34. Nesse contexto, um trabalho utilizando dados da população norte-americana mostrou que os gastos em saúde via desembolso direto aumentaram em função do crescimento do número de doenças crônicas dos moradores dos domicílios, mesmo após ajuste para as variáveis sociodemográficas e acesso a seguro de saúde privado. Além disso, famílias com indivíduos com doenças crônicas foram 2,6 vezes mais propensas a gastar mais que US\$ 1.000 anuais com assistência médica ${ }^{34}$. Esses achados são relevantes na medida em que a obesidade está associada à ocorrência de diversas doenças crônicas 5,6,7.

De modo geral, os domicílios brasileiros com indivíduos com excesso de peso e com obesos em sua composição apresentaram maiores gastos com a aquisição de medicamentos, resultado corroborado pela literatura científica, e pagamento de planos de saúde, mas não com os outros componentes da assistência à saúde, como internação, consultas e exames. No caso dos gastos com internação, a diferença de valores observada entre domicílios sem nenhum obeso em sua composição e com três ou mais indivíduos obesos, que representa $1 \%$ dos domicílios estudados, não foi estatisticamente confirmada. Analisando-se os gastos com internação no grupo de domicílios com três ou mais obesos, veri- 
Tabela 4

Valores preditos dos gastos familiares em saúde mensais (medicamentos e assistência à saúde), de acordo com o tipo de gasto, segundo o número de indivíduos com excesso de peso e obesidade nos domicílios brasileiros. Pesquisa de Orçamentos Familiares, Brasil, $2008-2009$.

\begin{tabular}{|c|c|c|c|c|c|c|c|c|c|c|c|}
\hline \multirow[t]{3}{*}{ Variáveis } & \multicolumn{11}{|c|}{ Gastos familiares em saúde mensais (Reais) * } \\
\hline & \multicolumn{4}{|c|}{ Medicamentos } & \multicolumn{6}{|c|}{ Assistência à saúde } & \multirow[t]{2}{*}{ Total } \\
\hline & $\begin{array}{l}\text { Para } \\
\text { doenças } \\
\text { crônicas }\end{array}$ & $\begin{array}{l}\text { Para doenças } \\
\text { infecciosas }\end{array}$ & Outros & Total & $\begin{array}{c}\text { Planos } \\
\text { de saúde }\end{array}$ & Internações & Consultas & Exames & Outros & Total & \\
\hline \multicolumn{12}{|l|}{$\begin{array}{l}\text { Indivíduos } \\
\text { com excesso } \\
\text { de peso nos } \\
\text { domicílios }\end{array}$} \\
\hline Nenhum & 14,78 & 15,37 & 10,33 & 40,48 & 70,68 & 21,79 & 16,79 & 0,52 & 0,41 & 110,18 & 150,67 \\
\hline 1 & 20,26 & 16,24 & 11,02 & 47,53 & 86,72 & 21,06 & 19,46 & 0,64 & 2,06 & 129,95 & 177,47 \\
\hline 2 & 26,57 & 19,43 & 12,24 & 58,25 & 112,70 & 28,24 & 22,59 & 0,79 & 1,18 & 165,50 & 223,75 \\
\hline 3 ou mais & 29,24 ** & 20,62 ** & $12,55 * \star *$ & $62,41 * \star$ & 134,01 ** & 52,82 & 17,14 & 0,55 & 1,77 & 206,29 ** & 268,70 ** \\
\hline \multicolumn{12}{|l|}{$\begin{array}{l}\text { Indivíduos } \\
\text { obesos nos } \\
\text { domicílios }\end{array}$} \\
\hline Nenhum & 18,81 & 16,31 & 10,92 & 46,04 & 90,44 & 21,72 & 19,27 & 0,59 & 1,46 & 133,48 & 179,52 \\
\hline 1 & 26,05 & 19,24 & 12,64 & 57,93 & 98,52 & 29,16 & 19,87 & 0,67 & 0,86 & 149,08 & 207,01 \\
\hline 2 & 34,83 & 21,46 & 11,05 & 67,34 & 128,27 & 34,38 & 16,93 & 0,98 & 3,04 & 183,61 & 250,95 \\
\hline 3 ou mais & 29,88 ** & 21,40 ** & 10,14 & $61,41 * \star$ & $\begin{array}{c}151,25 \\
\star \star \star\end{array}$ & 297,39 & 17,21 & 0,97 & 1,49 & 468,31 & 529,72 *** \\
\hline
\end{tabular}

* Valores ajustados para região, área, renda domiciliar per capita, presença de idosos e número de moradores nos domicílios;

** $p<0,001$ em modelo de regressão linear ajustado;

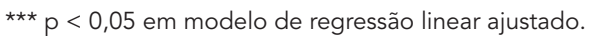

ficou-se uma grande variação, de nenhum gasto a $\mathrm{R} \$ 41.200,00$

Destaca-se que os gastos com assistência à saúde são fortemente influenciados pela condição social da família, pelo acesso a planos de saúde e pelo uso do SUS para procedimentos de alto custo, o que pode explicar a sua não associação com a obesidade.

Foi observado que $69 \%$ dos domicílios sem plano de saúde referiram algum gasto com assistência à saúde, com um dispêndio médio de $\mathrm{R} \$$ 40,08 , enquanto entre os domicílios que tinham plano de saúde, apenas $31 \%$ apresentaram algum gasto com assistência (em média $\mathrm{R} \$ 105,89$, excluindo o valor pago pelo plano de saúde). Assim, uma maior proporção de domicílios sem plano de saúde apresentou gastos com assistência à saúde, mas sendo um valor reduzido, por não terem recursos suficientes ou por utilizarem o SUS. Entre os domicílios que tinham plano de saúde, uma menor proporção apresentou gastos adicionais, no entanto, aqueles que tiveram, registraram maiores gastos, em função do tipo de plano de saúde que possuíam ou por poderem gastar com benefícios adicionais. Para os procedimentos de alto custo, provavelmente, mesmo quem tem plano de saúde utiliza o SUS.

De acordo com a OMS, a importância dos sistemas nacionais de saúde não está relacionada apenas com as melhorias nas condições de vida da população, mas também com a proteção contra o custo financeiro das doenças. Dessa forma, o desafio para os governos é reduzir a carga regressiva dos gastos privados em saúde, decorrentes de desembolso direto e de pagamento de planos de saúde, ou seja, as famílias mais pobres devem comprometer menor parte de sua renda com este tipo de gasto, o inverso acontecendo com a população mais rica, a fim de reduzir o gasto catastrófico com saúde 35 .

No presente trabalho, observou-se que a população mais pobre, em relação àquela com maior renda, comprometeu uma maior parte de sua renda com saúde (6\% contra 3,4\%). Gastos familiares em saúde têm sido considerados catastróficos quando ultrapassam $10 \%$ ou mais da 
renda, mas revisões da literatura para estudos sobre consequências econômicas de doenças e gastos familiares em saúde apresentam resultados semelhantes aos encontrados neste estudo, para países de renda média ou baixa 12,19 , sendo estes valores também semelhantes aos registrados em trabalhos realizados em países com renda alta 30 . Estudo realizado com os dados das POF de 2002-2003 e 2008-2009 utilizou diferentes pontos de corte para a definição de pobreza, e constatou que os gastos em saúde contribuem para que um maior número de domicílios seja classificado como pobre, com importante contribuição dos gastos em medicamentos 36 .

A comparação entre os estudos de gastos em saúde nos domicílios entre os diferentes países e a definição do que são gastos catastróficos apresenta dificuldades metodológicas importantes, face às diferenças nas formas de medir os gastos (diretos e/ou indiretos, referidos ou medidos diretamente, por meio de sistemas de informação administrativos, prospectivos ou retrospectivos etc.) e na definição do indicador utilizado para medir gastos catastróficos (proporção da renda domiciliar e/ou proporção dos gastos em saúde entre os gastos familiares, descontados ou não os gastos com alimentação). O indicador "proporção dos gastos familiares em saúde/renda familiar" ainda é muito utilizado, mas tem como restrição importante o fato da renda ser inelástica, isto é, não se expande quando sob pressão, o que faz com que as famílias não gastem o necessário para o enfrentamento do problema de saúde, se endividem ou vendam bens 37 .

Este estudo utilizou dados de pesquisa de orçamentos familiares e, portanto, apresenta limitações inerentes a estes dados. Na POF, as informações sobre despesas com medicamentos e assistência à saúde se referem ao período de 30 e 90 dias anteriores à data da pesquisa, respectivamente. Dessa forma, gastos decorrentes de doenças agudas e sazonais, acidentes, cirurgias de emergência e outros eventos instáveis podem ter sido subestimados no inquérito. No entanto, as condições crônicas, com maior chance de terem sido relatadas, devem estar mais fortemente associadas à obesidade. Ainda, há a possibilidade de sub-relato das despesas pelos indivíduos. Devido a tais limitações, acredita-se que os resultados podem estar subestimados e os gastos familiares em saúde nos domicílios brasileiros serem maiores que o verificado.

Outra limitação está relacionada ao fato do questionário que contém os dados sobre os gastos em saúde ter sido preenchido apenas pelos indivíduos com mais de 10 anos residentes nos domicílios amostrados. Em função disso, não é possível conhecer a distribuição intradomiciliar dos gastos, tendo em vista que pode ocorrer a situação de um indivíduo comprar medicamentos ou realizar o pagamento das despesas com assistência à saúde para outros membros do domicílio ou mesmo para as crianças menores de 10 anos. Nesse sentido, e considerando que a dinâmica familiar afeta a decisão sobre as despesas, optou-se por considerar o domicílio, e não o indivíduo, como a unidade de análise e utilizar como variáveis de interesse os gastos mensais em saúde e a contagem de indivíduos com excesso de peso e obesos nos domicílios.

A despeito de tais limitações, a POF 20082009 coletou dados de grande amostra (55.970 domicílios) e permite a obtenção de estimativas robustas para o país, suas cinco regiões, domicílios situados nas áreas urbana e rural e todas as faixas de renda 23 . Essa é uma importante vantagem de se utilizar pesquisas de orçamentos familiares, uma vez que tal cenário não é facilmente reproduzido em inquéritos de saúde, devido ao alto custo e à dificuldade de operacionalização da coleta. Ainda, merece menção o fato das medidas antropométricas terem sido aferidas e não referidas, como ocorre em outros estudos de custo da obesidade 33,38 .

Conclui-se que o excesso de peso e a obesidade influenciaram positivamente os gastos privados em saúde das famílias brasileiras, uma vez que a presença e o aumento do número de indivíduos com excesso de peso e obesidade nos domicílios resultaram em maiores gastos com medicamentos e planos de saúde. Acredita-se que para avaliar o impacto da obesidade para o país é necessário utilizar uma combinação de dados que envolvem aqueles oriundos dos sistemas de informação do SUS, como o Sistema de Informações Hospitalares e o Sistema de Informações Ambulatoriais do SUS, informações sobre a atenção básica e também pesquisas nos moldes da POF, capazes de mensurar os gastos privados em saúde. 


\section{Resumen}

El objetivo del estudio fue evaluar la influencia del exceso de peso y obesidad en el gasto en salud en los hogares brasileños. Se utilizaron los datos de la Encuesta de Presupuestos Familiares de 2008-2009 para estimar los gastos en salud, que contiene los gastos con la compra de medicinas y el gasto con los servicios de atención médica privada. También se evaluó el estado nutricional de las personas de los 55.970 hogares. El gasto mensual privado por persona en salud y sus componentes fueron analizados de acuerdo con la cantidad en número de personas con exceso de peso y obesidad en los hogares estratificados en cuatro categorías (ninguna, una, dos y tres o más personas). La presencia y el aumento en el número de individuos con exceso de peso y obesidad en los hogares resultaron en un mayor gasto en salud, especialmente el gasto en medicinas y seguros de salud privados. Estos resultados se mantuvieron después de realizar el ajuste pertinente. El exceso de peso y la obesidad influenciaron positivamente el gasto en salud de las familias brasileñas.

Obesidad; Gastos en Salud; Medicamentos;

Prestación de Atención de Salud

\section{Referências}

1. World Health Organization. Diet, nutrition and the prevention of chronic diseases. Geneva: World Health Organization; 2003. (WHO Technical Report Series, 916).

2. Finucane MM, Stevens GA, Cowan MJ, Danaei G, Lin JK, Paciorek CJ, et al. National, regional, and global trends in body-mass index since 1980: systematic analysis of health examination surveys and epidemiological studies with 960 countryyears and 9,1 million participants. Lancet 2011; 377:557-67.

3. Instituto Brasileiro de Geografia e Estatística. Pesquisa de Orçamentos Familiares 2008-2009 antropometria e estado nutricional de crianças, adolescentes e adultos no Brasil. Rio de Janeiro: Instituto Brasileiro de Geografia e Estatística; 2010.

\section{Colaboradores}

D. S. Canella participou da concepção e do delineamento do estudo, fez as análises dos dados, redigiu a versão inicial do manuscrito e revisou e aprovou a versão final. H. M. D. Novaes e R. B. Levy participaram da concepção e do delineamento do estudo e revisaram e aprovaram a versão final.

\section{Agradecimentos}

Este trabalho integra um projeto financiado pela Fundação de Amparo à Pesquisa do Estado de São Paulo (processo número 2012/17977-4).
4. Withrow D, Alter DA. The economic burden of obesity worldwide: a systematic review of the direct costs of obesity. Obesity Rev 2011; 12:131-41.

5. Sichieri R, Nascimento S, Coutinho W. The burden of hospitalization due to overweight and obesity in Brazil. Cad Saúde Pública 2007; 23:1721-7.

6. Bahia L, Coutinho ESF, Barufaldi LA, Abreu GA, Malhão TA, Souza CPR, et al. The costs of overweight and obesity-related diseases in the Brazilian public health system: cross-sectional study. BMC Public Health 2012; 12:440.

7. Oliveira ML, Santos LMP, Silva EN. Direct healthcare cost of obesity in Brazil: an application of the cost-of-illness method from the perspective of the public health system in 2011. PLoS One; 2015; 10:e0121160. 
8. Paim J, Travassos C, Almeida C, Bahia L, Macinko J. The Brazilian health system: history, advances, and challenges. Lancet 2011; 377:1778-97.

9. Porto SM, Ugá MAD, Moreira RS. Uma analise da utilização de serviços de saúde por sistema de financiamento: Brasil, 1998-2008. Ciênc Saúde Coletiva $2011 ; 16: 3795-806$.

10. Instituto Brasileiro de Geografia e Estatística. Contas Nacionais - conta-satélite de saúde. Brasil, 2007-2009. Rio de Janeiro: Instituto Brasileiro de Geografia e Estatística; 2012.

11. Xu K, Evans DB, Kawabata K, Zeramdini R, Klavus J, Murray CJ. Household catastrophic health expenditure: a multicountry analysis. Lancet 2003 ; 362:111-7.

12. McIntyre D, Thiede M, Dahlgren G, Whitehead M. What are the economic consequences for households of illness and of paying for health care in low- and middle-income country contexts? Soc Sci Med 2006; 62:858-65.

13. Barros AJD, Bastos JL, Dâmaso AH. Catastrophic spending on health care in Brazil: private health insurance does not seem to be the solution. Cad Saúde Pública 2011; 27 Suppl 2:S254-62.

14. Fang K, Ma C, Jiang Y, Ye L, Shia B, Ma S. Illness, medical expenditure and household consumption: observations from Taiwan. BMC Public Health 2013; 13:743.

15. Rahman MM, Gilmour S, Saito E, Sultana P, Shibuya K. Health-related financial catastrophe, inequality and chronic illness in Bangladesh. PLoS One 2013; 8:e56873.

16. Garcia LP, Santanna AC, Freitas LRS, Magalhães LCG. Gastos com saúde das famílias brasileiras: um recorte regional a partir das Pesquisas de Orçamentos Familiares 2002-2003 e 2008-2009. In: Boueri R, Costa MA, organizadores. Brasil em desenvolvimento 2013: estado, planejamento e políticas públicas. v. 3. Brasília: Instituto de Pesquisa Econômica Aplicada; 2013. p. 859-76.

17. Yusuf F, Leeder SR. Can't escape it: the out-ofpocket cost of health care in Australia. Med J Aust 2013; 199:475-8.

18. Thorpe KE, Florence CS, Joski P. Which medical conditions account for the rise in health care spending? Health Aff (Millwood) 2004; Suppl Web Exclusives:W4-437-45.

19. Kankeu HT, Saksena P, Xu K, Evans DB. The financial burden from non-communicable diseases in low- and middle-income countries: a literature review. Health Res Policy Syst 2013; 11:31.

20. Sichieri R, Coitinho DC, Leão MM, Recine E, Everhart JE. High temporal, geographic, and income variation in body mass index among adults in Brazil. Am J Public Health 1994; 84:793-8.

21. Monteiro CA, Conde WL, Popkin BM. Income-specific trends in obesity in Brazil: 1975-2003. Am J Public Health 2007; 97:1808-12.

22. Chukwuonye II, Chuku A, Okpechi IG, Onyeonoro UU, Madukwe OO, Okafor GO, et al. Socioeconomic status and obesity in Abia State, South East Nigeria. Diabetes Metab Syndr Obes 2013; 6:371-8.

23. Instituto Brasileiro de Geografia e Estatística. Pesquisa de Orçamentos Familiares 2008-2009 - despesas, rendimentos e condições de vida. Rio de Janeiro: Instituto Brasileiro de Geografia e Estatística; 2010 .
24. Silveira FG, Osório RG, Piola SF. Os gastos das famílias com saúde. Ciênc Saúde Coletiva 2002; 7:719-31.

25. Cerveny CMO. A família como modelo: desconstruindo a patologia. São Paulo: Editora Livro Pleno; 2000.

26. World Health Organization. WHO child growth standards: length/height-for-age, weight-for-age, weight-for-length, weight-for-height and body mass index-for-age: methods and development. Geneva: World Health Organization; 2006.

27. de Onis M, Onyango AW, Borghi E, Siyam A, Nishida C, Siekmann J. Development of a WHO growth reference for school-aged children and adolescents. Bull World Health Organ 2007; 85:660-7.

28. World Health Organization. Physical status: the use and interpretation of anthropometry. Geneva: World Health Organization; 1995. (Technical Report Series, 854)

29. Veiga P. Out-of-pocket health care expenditures due to excess of body weight in Portugal. Econ Hum Biol 2008; 6:127-42.

30. Waters HR, Anderson GF, Mays J. Measuring financial protection in health in the United States. Health Policy 2004; 69:339-49.

31. Raebel MA, Malone DC, Conner DA, Xu S, Porter JA, Lanty FA. Health services use and health care costs of obese and nonobese individuals. Arch Intern Med 2004; 164:2135-40.

32. Wee CC, Phillips RS, Legedza ATR, Davis RB, Soukup JR, Colditz GA, et al. Health care expenditures associated with overweight and obesity among US adults: importance of age and race. Am J Public Health 2005; 95:159-65.

33. Tarride JE, Haq M, Taylor VH, Sharma AM, NakhaiPour HR, O'Reilly D, et al. Health status, hospitalizations, day procedures, and physician costs associated with body mass index (BMI) levels in Ontario, Canada. Clinicoecon Outcomes Res 2012; 4:21-30.

34. Hwang W, Weller W, Ireys H, Anderson G. Out-ofpocket medical spending for care of chronic conditions. Health Aff (Millwood) 2001; 20:267-78.

35. World Health Organization. The World Health Report 2000. Health systems: improving performance. Geneva: World Health Organization; 2000.

36. Boing AC, Bertoldi AD, Garcia LP, Peres KG. Influência dos gastos em saúde no empobrecimento de domicílios no Brasil. Rev Saúde Pública 2014; 48:797-807.

37. Goryakin Y, Suhrcke M. The prevalence and determinants of catastrophic health expenditures attributable to non-communicable diseases in lowand middle-income countries: a methodological commentary. Int J Equity Health 2014; 13:107.

38. Finkelstein MM. Obesity, cigarette smoking and the cost of physicians' services in Ontario. Can J Public Health 2001; 92:437-40.

Recebido em 09/Dez/2014

Versão final reapresentada em 31/Mar/2015

Aprovado em 11/Mai/2015 